\title{
Regard sur vingt ans de travaux : le Journal of the
} History of Sexuality

Twenty years of scholarship in the Journal of the History of Sexuality

\section{Robert Nye}

\section{(2) OpenEdition \\ 1 Journals}

Édition électronique

URL : http://journals.openedition.org/clio/9693

DOI : $10.4000 /$ clio.9693

ISSN : $1777-5299$

\section{Éditeur}

Belin

Édition imprimée

Date de publication : 1 mai 2010

Pagination : 239-266

ISSN : 1252-7017

\section{Référence électronique}

Robert Nye, "Regard sur vingt ans de travaux : le Journal of the History of Sexuality », Clio. Femmes,

Genre, Histoire [En ligne], 31 | 2010, mis en ligne le 31 mai 2010, consulté le 21 avril 2019. URL : http:// journals.openedition.org/clio/9693; DOI : 10.4000/clio.9693 


\section{Regard sur vingt ans de travaux : le Journal of the History of Sexuality}

Robert NYE

Les universitaires français le savent: l'histoire de la sexualité est née dans les universités anglo-américaines ${ }^{1}$. Ce qui peut s'expliquer en partie par la puissance et l'audace des mouvements de libération sexuelle des années 1960 et 1970, qui revendiquaient le droit à une expression sexuelle plus libre ainsi que l'abrogation des lois qui contrôlaient les pratiques sexuelles des gay et des lesbiennes. Cependant, lorsque, au début des années 1980, on vit se développer un enseignement autour de ces questions, celui-ci s'appuya également sur l'autonomie et la flexibilité des institutions universitaires angloaméricaines. Il faut aussi faire la part de l'apparition concomitante de départements d'études interdisciplinaires consacrés aux femmes (et aux hommes) et au développement des études culturelles (cultural studies) qui se sont consacrées à l'exploration des moyens par lesquels on a opprimé les minorités sexuelles, religieuses, ethniques et raciales dans le passé. Ainsi, l'histoire de la sexualité a été conçue dans un contexte de revendications politiques que ses acteurs n'ont jamais cherché à nier, au point qu'elle en porte encore les traces - en dépit d'une maturation certaine, de la création de revues et collections spécialisées et de son intégration dans les cursus universitaires.

Fondé en 1990, le Journal of the History of Sexuality fut le premier périodique à être entièrement consacré à l'histoire de la sexualité. GLQ, Sexualities et Studies in Gender and Sexuality, qui sont toutes des revues qui ont vu le jour plus tard, dans les années 1990, publient souvent des articles d'historiens mais, la plupart du temps, ces revues

1 Rebreyend 2005 ; Fassin 2004. 
sont de nature plus interdisciplinaire et affichent un parti pris plus explicite que le JHS. L'historien John C. Fout, qui fut le premier rédacteur en chef du JHS, expliquait dans le numéro un que le but de la revue était d'extraire l'étude de la sexualité du domaine médical de la sexologie pour inciter à un fonctionnement "transdisciplinaire » associant «historiens des sciences sociales, sociologues, anthropologues, philosophes, psychologues, chercheurs en littérature, spécialistes de l'Antiquité, historiens de l'art et du cinéma... $»^{2}$. On le voit, il n'accordait pas une attention particulière à l'histoire, pour la simple et bonne raison que très peu d'historiens travaillaient sur l'histoire de la sexualité à cette époque: on aurait pu compter les cours de Licence sur les doigts d'une main et il n'existait aucune formation doctorale sur le sujet. Si elle voulait se développer encore, la jeune revue allait devoir compter sur des spécialistes issus d'autres domaines. John C. Fout indiquait aussi que le JHS deviendrait un forum international qui publierait des articles d'auteurs du monde entier et proposerait des comptes rendus d'ouvrages dans de nombreuses langues. Par la suite, aucune de ces promesses d'orientation éditoriale ne fut réellement tenue. La revue est devenue un périodique d'histoire, dont les collaborateurs sont presque tous des spécialistes de ce domaine et, bien qu'elle publie des articles sur la sexualité telle qu'elle existe ou a existé dans le monde entier et durant toutes les époques historiques, tous les comptes rendus de lecture portent sur des ouvrages en anglais, à quelques exceptions près, tandis que ses collaborateurs travaillent tous en Amérique du Nord et en Europe. La revue possède une version électronique en ligne depuis 2001 et on peut y accéder sur le portail du Projet Muse.

Malgré ces évolutions, personne ne peut dire que le JHS s'est replié sur lui-même ou qu'il est devenu absolument inaccessible aux non-spécialistes. Les rédacteurs en chef successifs n'ont publié aucun manifeste, ils n'ont pris aucune position politique particulière mais, comme nous le verrons, ils restent fidèles aux origines politiques progressistes des études contemporaines sur la sexualité puisqu'ils publient des travaux sur l'actualité des politiques sexuelles, donnent l'occasion à des spécialistes de débattre de questions controversées et

2 Fout $1990: 1$. 
accordent un espace d'expression important au potentiel de radicalité qu'induit l'historicisation. La définition classique de cette fonction critique de l'analyse historique a été exposée de manière éloquente par Michel Foucault, dont l'influence sur l'histoire de la sexualité reste inégalée à ce jour :

Ce que la raison éprouve comme sa nécessité, ou ce que plutôt les différentes formes de rationalité donnent comme leur étant nécessaire, on peut parfaitement en faire l'histoire et retrouver les réseaux de contingences d'où cela a émergé. (...) Cela veut dire qu'elles reposent sur un socle de pratique humaine et d'histoire humaine et, puisque ces choses-là ont été faites, elles peuvent, à condition qu'on sache comment elles ont été faites, être défaites ${ }^{3}$.

Les collaborateurs du JHS ont systématiquement adopté cette perspective critique dans leurs travaux, en particulier dans leurs recherches sur la médicalisation de la sexualité, sur la création et le renforcement des stéréotypes de genre et de sexe, et sur l'impact de la religion et de la théologie sur les normes sexuelles.

Je diviserai ma présentation en trois sections: domaines d'investigation, thèmes et méthodes. Ces distinctions sont arbitraires : on pourrait imaginer d'autres façons d'analyser les contenus de la revue depuis vingt ans environ qu'elle existe. Je veux mettre ici l'accent sur le développement de l'histoire de la sexualité en tant que champ d'études qui a des liens profonds avec ce qui intéresse les lecteurs de la revue CLIO: l'histoire des femmes, le genre, le féminisme et la sexualité féminine. Hommes et masculinité figureront également dans mon analyse. Que l'on considère le genre comme une construction d'idéaux culturels ou comme une expérience vécue, personne ne met en doute la persistance historique d'un système binaire fondé sur l'existence de corps masculins et féminins, d'un comportement genré et de représentations culturelles liées au genre qui s'influencent mutuellement. Le genre façonne la sexualité par l'intermédiaire de normes et de pratiques qui ont aussi marqué les relations sexuelles entre personnes de même sexe et qui ont reçu à travers l'histoire l'imprimatur des élites scientifiques, médicales et religieuses. Je n'emploie pas ici le mot "genre" (gender) comme un

3 Foucault 1988 : 37 ; en français dans Foucault 1994 : 449. 
synonyme de "sexe". Joan Scott l'a signalé, il y a longtemps : «le genre est une catégorie sociale que l'on impose à un corps sexué $»^{4}$. J'ai expliqué ailleurs que les corps sexués avaient aussi leur propre histoire, que ces histoires ont un rapport avec le genre mais ne lui sont pas consubstantiels ${ }^{5}$. En France, aujourd'hui, l'emploi du mot "genre" est beaucoup plus fréquent qu'il ne l'était à la naissance de la revue CLIO mais, comme Karen Offen l'a montré, des termes, qui avaient des sens très proches, circulaient en France dès l'époque moderne ${ }^{6}$.

\section{Domaines d'investigation : espace/temps}

Les champs géographiques couverts par le JHS ont surtout été limités à l'Europe et à l'Amérique du Nord. Un peu plus de trente articles seulement se sont intéressés à d'autres parties du monde et au moins une douzaine d'entre eux ont exploré des questions de prostitution, de race ou de réglementation de la sexualité dans les colonies européennes ou les territoires conquis. Depuis 2000, un certain nombre de numéros thématiques ont traité de questions sexuelles en Amérique du Nord ou dans les États européens, mais un seul numéro s'est penché sur ces questions telles qu'elles se manifestent dans d'autres parties du monde ${ }^{7}$. Parfois, la revue exprime le souhait que des sujets soient abordés dans une perspective d'histoire globale mais, jusqu'ici, les anthropologues et autres chercheurs en sciences sociales se sont montrés plus disposés et plus compétents que les historiens pour entreprendre de tels travaux ${ }^{8}$ La plupart du temps, les historiens sont formés pour devenir spécialistes d'histoire nationale ou régionale. Cela changera sans aucun doute mais, actuellement, la recherche sur la sexualité dans le monde est loin de pouvoir rendre compte du redécoupage de la géographie sexuelle qui accompagne le phénomène de la mondialisation.

\footnotetext{
4 Scott $1988: 32$.

5 Nye 2008.

6 Thébaud \& Zancarini-Fournel 2002 : 4 ; Offen 2006.

7 JHS 2007, vol. 16, 3, sur l'Amérique latine.

$8 \quad$ Rupp 2001 ; voir Bleys 1996 ; Altman 2001.
} 
Ne pas tenir compte de l'histoire des sexualités non occidentales présente un risque indéniable car cela encourage les chercheurs à élaborer des modèles de sexualité entièrement fondés sur cette expérience occidentale, à partir desquels d'“autres" sexualités croisées à l'occasion de voyages ou dans les colonies seront décrites comme des comportements anormaux dus aux conditions matérielles et morales propres à telle ou telle société "primitive". Certains collaborateurs du JHS qui travaillent sur l'impérialisme européen ont expliqué cette tendance en montrant comment les sexualités indigènes ont été interprétées, jugées et, plus particulièrement, nommées selon les critères européens du comportement sexuel et de la décence propres à chaque genre ${ }^{9}$. Lorsqu'on les plaquait sur des cultures indigènes, les concepts occidentaux historiques de pédérastie, d"“amours saphiques", de "vice contre nature", d'homosexualité et d'effémination se révélaient, pour les colonisateurs, de dangereuses formes de corruption qui menaçaient tout le projet colonial, d'autant plus si elles étaient courantes. Dans son étude sur l'identité transgenre et sur le comportement sexuel entre hommes en Thailande, l'historien australien Peter A. Jackson montre que les cultures occidentales n'ont jamais su interpréter ces phénomènes et il explique comment le cas thailandais, parallèlement à d'autres exemples, révèle les «limites culturelles de l'analyse foucaldienne ${ }^{10} »$.

En revanche, le $J H S$ a régulièrement abordé toutes les périodes de l'histoire occidentale, de l'Antiquité grecque à nos jours. Les sociétés grecques et romaines anciennes, ainsi que l'Europe médiévale, ont bénéficié d'une attention toute particulière. Dans les départements universitaires de Lettres classiques et d'Études médiévales, ces époques jouissent depuis longtemps d'une espèce d'autonomie; il n'est donc pas surprenant qu'elles aient suscité d'intéressants débats sur l'histoire de la sexualité. Michel Foucault d'abord, puis David Halperin et John J. Winkler ont avancé l'idée que les relations entre adultes de même sexe ne jouaient aucun rôle dans la culture classique, dans la mesure où celle-ci était plutôt régie par un modèle de

$9 \quad$ Levine 1994 ; Spaulding \& Beswick 1995 ; Manderson 1997 ; Sigal 1997 ; Forman 2002 ; Proschan 2002.

10 Jackson 1997 ; voir aussi Penrose 2001 ; Sweet \& Zwilling 1993. 
pénétration dominant/dominé favorisant les relations pédérastiques entre adultes, mineurs et "autres" catégories sexuelles telles que les esclaves"1. De ce point de vue, on a pu dire que "l'homosexualité" avait pris son essor à la fin du XIX siècle, au moment même où naissait ce nouveau mot. Amy Richlin et Rabun Taylor ont contesté cette idée, affirmant à l'inverse qu'il existait déjà dans la Rome antique des mots approchants pour désigner l'amour entre adultes du même sexe, ainsi qu'une sous-culture "homosexuelle" refoulée qui allait de pair avec un vif sentiment d'identité "homosexuelle"12.

Les questions d'identité et de continuité ont également fait l'objet d'un forum consacré au livre de Carolyn Dinshaw, Getting Medieval: Sexualities and Communities, Pre-and Post-Modern (1999), qui se penche sur la sexualité entre adultes de même sexe. Comme Richlin et Taylor sur la culture classique, Dinshaw, ainsi que la plupart des collaborateurs dudit forum, conteste en partie Michel Foucault et sa thèse des ruptures discursives fondamentales dans l'expérience historique, en soulignant l'existence de connexions entre l'histoire "queer", les historiens "queer", les sujets de l'histoire "queer", ainsi que les lecteurs "queer", tant et si bien que les frontières habituelles entre passé et présent, auteur et lecteur ${ }^{13}$ ont tendance à disparaitre. Même s'ils ont été moins retentissants, d'autres articles sur l'histoire médiévale font également ressortir des continuités, notamment entre l'ascétisme païen, la dimension anti-familiale du christianisme naissant ${ }^{14}$ et enfin la reconnaissance par la théologie et la société médiévale de deux fonctionnements bien distincts, le mariage et le célibat du clergé15. L'autre continuité longtemps entretenue par le christianisme est celle d'une peur sous-jacente de la sexualité non reproductive et, plus particulièrement, d'une sexualité féminine totalement débridée. L'Église et les pères de l'Église s'accordaient à dire que la sexualité et l'identité de la femme étaient rapportées à sa

\footnotetext{
11 Foucault 1986.

12 Richlin 1993; Taylor 1997 ; voir aussi le numéro thématique sur l'Antiquité tardive, JHS 2001, vol. 10, $3 / 4$.

13 JHS 2001, vol. 10, 2.

14 Clark E. 1995.

15 Clark C. 2007 ; Barco 2008 ; Wertheimer 2006.
} 
fertilité16. Cependant, selon Daniel Boyarin, les sources bibliques et talmudiques sur lesquelles s'appuient ces développements médiévaux confortent l'idée foucaldienne selon laquelle les Anciens ne connaissaient pas de "sexualité" autonome et que l'homosexualité ne constituait pas une identité ; la culture juive ancienne proscrivait tout comportement qui s'écartait des normes traditionnelles genrées, mais pas des normes sexuelles ${ }^{17}$.

L'Europe moderne est également bien représentée dans la revue mais l'époque contemporaine et, plus précisément, l'histoire britannique et américaine est particulièrement privilégiée. Bon nombre d'articles abordent des questions sexuelles très récentes : les activités politiques des gay et lesbiennes, le HIV/SIDA, la politique en matière de mariage homosexuel et les sexualités dans la culture populaire $^{18}$. Mais il est difficile d'apporter un bon éclairage historique sur des événements qui datent d'il y a seulement quelques années ou qui, parfois, sont toujours d'actualité. Toutefois, les historiens dont les travaux concernent des événements totalement "passés" tirent indéniablement profit de la lecture d'articles sur les évolutions contemporaines, et la réciproque est incontestablement vraie. S'il y a une chose à retenir de Foucault, c'est que les généalogies sexuelles commencent dans le présent.

\section{Thèmes}

Anne-Claire Rebreyend a souligné avec pertinence que, dans le cadre de l'historiographie anglo-américaine, la sexualité est plutôt de nature politique, qu'elle procède de manière réflexive et qu'elle se préoccupe principalement de l'histoire de la construction des homosexualités; alors qu'en France, l'histoire des sexualités est plus narrative, plus détachée du champ politique (à quelques exceptions notables près) et davantage centrée sur la prostitution, l'avortement et la violence sexuelle, qui sont des facettes des cultures

\footnotetext{
16 Karras 1990 ; Bitel 1992.

17 Boyarin 1995.

18 Irvine 1995; Hogeland 1995 ; Bell 1995; Adam 2003; Hannah-Jones 2003 ; Gilmore 1994. Ceci ne constitue qu'une petite partie des études existantes.
} 
hétérosexuelles ${ }^{19}$. Si l'on raisonne en termes de comparaison, il me semble que cette distinction est globalement juste et que, bien sûr, les raisons de cette divergence d'intérêt et de style sont ancrées dans les histoires nationales de chacun ${ }^{20}$. Cependant, il n'en demeure pas moins qu'il est impossible d'opposer radicalement les sexualités homosexuelles et hétérosexuelles comme étant deux catégories analytiques distinctes. Du point de vue conceptuel, elles se constituent l'une contre l'autre, dans un processus puisant à la fois des éléments masculins et féminins dans le système du genre qui, lui aussi, est dichotomique. Mais tout ceci porte sur l'histoire des concepts. À vrai dire, il n'existe pas d'identité sexuelle ou de genre qui soit pure et stable et qui ne soit altérée par son opposé ; il n'est pas d'individu pour qui le corps, les désirs, la présentation de soi et l'identité soient en parfaite adéquation, pas plus d'un point de vue ontologique que d'un point de vue temporel.

Néanmoins, le $J H S$ propose à ses lecteurs un ensemble cohérent de thèmes. L'histoire des gay et des lesbiennes est de loin le sujet le plus traité, avec peut-être une légère préférence pour l'homosexualité masculine. Dans presque chaque numéro de quatre ou cinq articles, un article au moins traite de l'un ou l'autre de ces sujets; deux numéros thématiques ont été consacrés à l'histoire des gay et des lesbiennes ou plutôt aux " histoires des gay et des lesbiennes »"21, l'un se concentrant sur l'histoire lesbienne ${ }^{22}$, l'autre sur l'homosexualité masculine dans l'Allemagne nazie ${ }^{23}$. Quelques articles abordent simultanément la question des relations homosexuelles des hommes et des femmes, mais surtout dans le contexte des réflexions théologiques ou juridiques sur les comportements anormaux de sexe et de genre ${ }^{24}$.

Les études consacrées aux lesbiennes et aux interprétations historiques de l'amour entre femmes sont extraordinairement riches

\footnotetext{
19 Rebreyend 2005.

$20 \quad$ Nye 2003 ; Eder \& Hall 1999.

21 JHS 1992, vol. 3, 2 et 1993, vol 4, 3.

22 JHS 2005, vol. 14, 1/2.

23 JHS 2008, vol. 17, 1.

24 Moeller 1994 ; Matysik 2004.
} 
et variées. Cependant, les débats sur le mot à utiliser pour désigner l'amour entre hommes durant l'Antiquité et jusqu'au début de la période contemporaine ainsi que sur les interdictions explicites à l'encontre de la sodomie (punie par la loi) expliquent que, mis à part les épisodes d'amours "tribade" dans les couvents ${ }^{25}$, les historiens ont concentré presque toute leur attention sur l'interdiction de l'amour entre hommes. Opérant une distinction qui fonctionne dans la plupart des sociétés de notre histoire, Robert G. Moeller a montré que, dans l'Allemagne contemporaine, sur le plan légal, la maternité a incarné l'essence et le rôle de la femme dans des proportions telles que, tant que la femme était fertile, on estimait qu'il n'y avait pas vraiment de raison de criminaliser ses penchants sexuels. À l'inverse, la loi considérait que les amours homosexuelles dissipaient le "pouvoir procréateur" des hommes et le réduisaient à une impuissance physiologique ou psychique - véritable crime à l'encontre de leur descendance et réel danger pour l'État populationniste ${ }^{26}$.

Avant l'époque contemporaine et l'apparition de l'adjectif "lesbienne" pour qualifier une identité personnelle, les veuves, les vieilles filles, les femmes d'une même famille et les femmes travaillant ensemble vivaient sous le même toit et partageaient le même lit dans un «monde féminin fait d'amour et de rituel $»^{27}$. Quelques articles du JHS ont étudié la manière dont les femmes ont construit leur identité individuelle et sexuelle à une époque où l'on ne parlait pas encore couramment de modèles d'amours "saphiques" ou lesbiens. Ces articles choisissent souvent la méthode de l'analyse textuelle, débusquant entre les lignes les "indéterminations" sexuelles, ou encore recherchant des personnages proto-lesbiens dans la littérature. $\mathrm{Au}$ fil de ses lectures, Anne Lister, une aristocrate britannique du début du XIX" siècle, découvre des "textes sexuels" romantiques à travers lesquels elle peut exprimer ses penchants ${ }^{28}$. À la fin du XIX siècle, les femmes qui gagnaient leur vie et vivaient ensemble dans

\footnotetext{
25 Rivers 1995.

26 Moeller 1994 : 403.

27 Smith-Rosenberg 1985.

28 Craft-Fairchild 2006 ; Meem 1997 ; Clark A. 1996.
} 
une forte relation affective avaient tout du couple marié, exception faite justement de cette appellation ; d'autres censuraient leur passion en s'adonnant à une expiation spirituelle de sentiments dont elles pensaient qu'ils étaient contre nature, ou peut-être tout simplement très peu fréquents ${ }^{29}$.

En 2000, la médiéviste Judith Bennett a proposé d'utiliser l'expression "apparenté lesbienne»(lesbian-like) pour permettre aux historiens du social de dépasser l'examen des discours pour étudier «en particulier les femmes dont la vie leur a permis de faire l'expérience d'amours lesbiennes, des femmes qui ont résisté aux codes de conduite féminine essentiellement fondés sur le mariage hétérosexuel, des femmes qui étaient en position de pouvoir aider ou soutenir d'autres femmes... ${ }^{30}$ ». L'exploration de ces différentes situations ne permet pas de dire si le désir homosexuel était alors encouragé ou simplement exprimé, mais elle permet de délimiter un terrain de l'histoire sociale. Vers la fin du XIXe siècle, le vocable "lesbien" devint plus fréquent, voire banal (comme quelques autres synonymes tels que "saphique"). Il est dès lors possible de voir comment le discours médical, la culture populaire et les réseaux féministes décrivaient le comportement et l'identité lesbiens. On ne peut pas dire que cette nouvelle circulation du mot forgea d'une manière ou d'une autre l'identité lesbienne mais elle servit sans nul doute de marqueur culturel et linguistique et eut de réels effets sociaux. Les sexologues commencèrent alors à étudier et à classer par catégories les perversions apparentées à ces comportements, y compris "l'inversion", qui renvoyait à l'orientation homosexuelle des hommes et des femmes. Ainsi, une grande partie de ce langage médical profondément désobligeant entra peu à peu dans la langue courante ${ }^{31}$.

Peut-être est-ce la raison pour laquelle, au début du siècle, la prudence empêchait les groupes féministes, en Allemagne tout au moins, de revendiquer la dépénalisation de l'homosexualité féminine,

29 Elias 2006 ; Phipps 2009.

30 Bennett 2000 : 9-10; voir l'utilisation de ce terme dans Amer 2009.

31 Bauer 2009 ; Beccalossi 2009. 
alors même qu'il devait y avoir des lesbiennes dans leurs rangs ${ }^{32}$. Anne Rüling, une féministe qui défendait le droit à l'homosexualité des femmes au nom des libertés individuelles, fit une déclaration en ce sens lors d'une assemblée organisée dans le cadre du Comité humaniste scientifique de Magnus Hirschfeld, et non lors d'une réunion féministe ${ }^{33}$. La scission entre féministes hétérosexuelles et lesbiennes semble avoir été maintenue jusqu'à un certain point pendant toute la seconde vague féministe. La crainte de voir les lesbiennes discréditer la cause du mouvement féministe classique entraîna des négociations sans fin entre lesbiennes et hétérosexuelles sur l'image du mouvement et la manière dont les objectifs féministes devaient être clairement exprimés ${ }^{34}$. À force d'être tenues à l'écart du débat, les lesbiennes du monde anglophone se mirent à tisser des liens communautaires forts: dans de nombreuses villes, elles formèrent leurs propres organisations, adoptèrent les modes de consommation de l'époque et furent à l'initiative de bon nombre d'activités culturelles et artistiques indépendantes ${ }^{35}$.

Ces évolutions indiquent que, sur le long terme, la formation des identités lesbiennes (et gay) est le résultat d'une interaction dialectique avec la société hétérosexuelle. Deborah Cohler a étudié la "construction" des lesbiennes dans la Grande-Bretagne de l'entredeux-guerres, qui s'élabora au cours de toute une série de batailles juridiques sur des questions de diffamation, de censure, d'accusations d'espionnage et de complots. Les procès et poursuites engagés contre les lesbiennes et les femmes qui affichaient des attitudes "saphiques" contribuèrent à façonner les identités lesbiennes dans cette situation de crise tout à fait exceptionnelle, rappelant d'une certaine manière ce qu'avait vécu la génération précédente au moment de la condamnation d'Oscar Wilde. Dans les deux cas, lesbiennes et homosexuels, désormais davantage conscients de la nécessité d'être

32 Matysik 2004.

33 Ibid.

34 Gilmore \& Kaminski 2007. Les conflits au sein du féminisme autour de la pornographie ont été également très vifs dans les années 1990. Voir Hogeland 1995 ; Bell 1995.

35 Freeman 2000 ; Murray 2007 ; Collard 2006. 
discrets, ont sans doute été plus profondément confortés dans leur identité, tandis que les hétérosexuels ont certainement réalisé qu'il pouvait être dangereux de s'écarter du droit chemin, ce qui les a confirmés dans leurs préjugés ${ }^{36}$.

La revue a publié un certain nombre d'articles sur la sexualité des femmes mais, à les lire, on en apprend probablement davantage sur la construction discursive de la sexualité féminine que sur la vie sexuelle des femmes à proprement parler. Rien de surprenant à cela. En matière de sexualité féminine, les figures opposées de la Madone et de la putain ont toujours dominé la pensée occidentale, qui a réussi à glisser entre les deux la figure de l'épouse (fertile). La crainte de l'escroquerie sexuelle a toujours joué un rôle dans le contrôle de la virginité des jeunes mariées; l'épreuve de la nuit de noces était décisive puisqu'elle devait attester de l'expérience masculine et de l'innocence féminine. Il n'y a guère que dans les écrits médicaux et les textes littéraires que l'on entend parler de "débâcles ${ }^{37}$. En effet, la littérature érotique, mais aussi le discours médical, les guides sur la sexualité et, plus récemment, la culture populaire ont façonné notre compréhension des pratiques et des désirs sexuels, sans exclure pour autant la sexologie "scientifique", de manière plus sûre que l'expérience ou les "faits" vécus par chacun ${ }^{38}$.

En France, au XVIII siècle, la littérature érotique était principalement écrite par et pour des hommes. On peut dire qu'elle a établi une distinction entre des jeunes femmes décrites comme naturellement «curieuses » de ce qu'est la sexualité et celles dont on disait qu'elles possédaient une nature sexuelle «innée» mais néanmoins «innocente» puisqu'elles ignoraient tout de ses fonctions ${ }^{39}$. Cette construction largement imaginaire, par ailleurs exclusivement destinée aux femmes issues des classes moyennes et de l'aristocratie, fut "validée" par la médecine telle qu'on la pratiquait en Europe et en Amérique au XIX siècle. Les perversions que les sexologues "découvrent" alors chez la femme (inversion, hystérie,

\footnotetext{
36 Cohler 2007 ; Erber 1996 ; voir aussi Gilmore 1994.

37 Gwilliam 1996 ; Cryle 2009.

38 Tiefer 1995 ; Robinson 1976 ; Bullough 1994.

39 Kraakman 1994.
} 
frigidité, nymphomanie et érotomanie) ne sont que le reflet des perversions sexuelles des hommes.

Ces déficiences ou excès de la fonction physiologique étaient interprétés dans le contexte d'un ordre naturel où un homme et une femme originels imaginaires déterminaient la morphologie physique, le genre et le désir sexuel ${ }^{40}$. Cependant, au cours de la seconde moitié du XIX ${ }^{e}$ siècle, la peur obsessionnelle de la dégénérescence humaine faisait craindre la disparition de la frontière entre les sexes, ce dont les perversions sexuelles étaient le symptôme. Chez la femme, lorsque les passions se détachaient du corps sexué "normal", cela se traduisait par des troubles déficitaires (hystérie, frigidité) ou par des excès (érotomanie, nymphomanie) ${ }^{41}$. De fait, dans ce paradigme médical, les crises d'identité sexuelle étaient aussi des crises du genre, indépendantes du sexe biologique des individus. Le modèle médical dominant estimait ainsi que les pathologies sexuelles de la femme étaient des anomalies de genre que l'on pouvait d'autant mieux guérir que l'on restaurait le genre "véritable"42. Ce n'est qu'au début du XXe siècle que Sigmund Freud remit en question les liens jusque-là intangibles entre sexe, genre et sexualité : loin d'être des anomalies d'un corps dégénéré, l'hystérie et les autres pathologies sexuelles de la femme étaient en fait des réponses traumatiques produites par le choc du réel sur une femme dont l'ignorance était prescrite - par les stéréotypes genrés de l'époque.

Les manuels sur la sexualité, l'hygiène et le mariage, qui s'efforçaient de combler le déficit de connaissance dans ces divers domaines, sont à porter au nombre des textes qui ont forgé nos représentations des désirs et des pratiques sexuelles des femmes. À mesure que régressait la conception orthodoxe de l'innocence sexuelle des femmes, un juste milieu s'établissait, concédant à la femme la recherche du plaisir dans le lit conjugal même si l'on estimait que l'initiative était toujours réservée à l'homme ${ }^{43}$. Ces

\footnotetext{
40 Worman 2009 ; Nye 2008.

41 Cryle \& Downing 2009 ; Bauer 2009 ; Moore 2009.

42 Pour le fonctionnement de ce processus à propos de l'anorexia nervosa, voir O’Connor 1995 ; pour l'addiction sexuelle, voir Irvine 1995.

43 Neuhaus 2000 ; Mesch 2009.
} 
évolutions ne remirent pas en cause les rôles genrés traditionnels; au contraire, elles étaient destinées à les renforcer en mettant sur le même plan plaisir sexuel, devoirs de la ménagère et fertilité. Le numéro thématique du JHS sur la sexualité et le fascisme allemand parvient à une conclusion similaire: le régime nazi était tout sauf puritain, même s'il associait désir sexuel et mariage "hygiénique", prostitution et citoyens productifs, sexualité en général et objectifs eugéniques de l'État ${ }^{44}$.

Les médias contemporains ont largement contribué à la fabrication des conceptions de la sexualité des femmes "modernes". Les médias les plus populaires, dont les magazines féminins, y participent quand ils évoquent la baisse de la chasteté prénuptiale, encourageant les femmes à profiter de cette évolution inéluctable pour optimiser leur plaisir sexuel, accroitre leurs relations ou encore faire avancer leur carrière professionnelle. Mais ces conseils sont invariablement liés à des représentations qui mettent l'accent sur la féminité, le charme sournois de la pudeur sexuelle et autres caricatures des femmes, dépeintes comme des objets manipulant le désir de l'homme ${ }^{45}$. Un article fascinant d'Ingrid Sharp sur «l'unification sexuelle » de l'Allemagne dans les années 1990 révèle qu'en Allemagne de l'Ouest, les médias ont fait grand cas de l'émancipation sexuelle et du plaisir personnel prétendument plus fréquents chez les femmes d'une RDA sécularisée. Une rumeur se répandit alors, selon laquelle ces femmes, de culture traditionnelle, étaient des «chaudes» et qu'elles allaient attirer les Allemands de l'Ouest (Wessis), plus riches et plus conquérants que les "femmelettes d'Ossis», ainsi détrônés du fait d'une culture estallemande qui les avait habitués à participer aux tâches ménagères et à l'éducation des enfants ${ }^{46}$.

Parfois, certains articles abordent deux thèmes conjointement : tel est le cas de la prostitution et de la médicalisation de la sexualité féminine pour lesquelles les auteurs s'appuient sur des sources littéraires, administratives et médicales. Le JHS a publié autant

\footnotetext{
44 Herzog 2002 ; Heinemann 2002 ; Gordon 2002.

45 Berebitsky 2006 ; Pitzulo 2008.

46 Sharp 2004.
} 
d'articles sur la prostitution coloniale que sur la réglementation de la prostitution en métropole. De nouvelles études de cas sur les origines des réglementations ont été publiées en Amérique du Nord et en Europe $^{47}$. Les travaux les plus intéressants sur la prostitution en Europe concernent les efforts des féministes allemandes au début du siècle pour obliger l'État à abolir le fonctionnement discriminatoire de la police ; les féministes abolitionnistes avaient d'ailleurs remporté semblable victoire quelques années plus tôt en Grande-Bretagne. Les efforts des féministes allemandes furent couronnés de succès en 1927, seulement après que ces féministes eurent adopté un discours médical qui permettait de transférer le contrôle de la prostitution des mains des fonctionnaires de police à l'institut d'hygiène publique. Mais, les droits des prostituées ne s'en trouvèrent guère améliorés ${ }^{48}$.

Comme il n'y avait (presque) pas d'opposition libérale ou féministe à la réglementation de la prostitution dans les régimes coloniaux japonais et européens, les solutions médicales aux "problèmes" de réglementation furent adoptées partout dans le monde à la fin du XIXe siècle. Ce fut le cas également dans les milieux militaires. Les féministes européennes ont tout de même pu contester la «féminisation des maladies vénériennes» en soulignant la responsabilité des hommes dans la propagation de la syphilis et autres infections sexuellement transmissibles alors qu'aux frontières coloniales et militaires, le comportement sexuel des femmes indigènes (et européennes) faisaient l'objet d'une surveillance morale et médicale particulièrement sévère ${ }^{49}$. Les efforts engagés pour contrôler la sexualité masculine étaient généralement battus en brèche par la conviction, qu'en mission, les hommes avaient besoin d'exutoires sexuels et que ce qu'il y avait de mieux à faire était de distribuer des préservatifs et de soumettre les prostituées à des examens médicaux ${ }^{50}$. La situation des femmes indigènes était surdéterminée par l'alliance des perspectives médicales sur la question sexuelle et sur la question

\footnotetext{
47 Gabbert 2003 ; Guereña 2008.

48 Allen 1993.

49 Spongberg 1998.

50 Kampf 2008 ; Timm 2002.
} 
raciale, qui trouvaient une combinaison particulière dans la figure de la prostituée ${ }^{51}$.

Comme nous l'avons vu plus haut, au XIXe siècle, l'autorité du modèle médical de santé s'étendait bien au-delà du traitement de la maladie organique pour englober tout phénomène psychique ou social pour lequel il y avait un consensus sur les pathologies et les normes. Il y a très longtemps déjà, Jacques Donzelot a expliqué que, dans la deuxième moitié du XIX siècle, la médecine a endossé un rôle de conseil auprès des familles et de la société en général, rôle traditionnellement joué par l'Église. Toutefois, les médecins n'ont pas vraiment réfuté la dimension morale du dogme chrétien : ils l'ont même plutôt intégrée à une perspective médicale matérialiste ${ }^{52}$. Par conséquent, on peut parler d'une orientation moralisatrice de la médecine scientifique. Les effets de ces évolutions ont été cruciaux pour les femmes. À la fin du XVIII ${ }^{e}$ siècle, les représentations traditionnelles de la sexualité robuste et pleine de vitalité de la population rurale ont évolué ; il est dorénavant question des dangers moraux et médicaux du vagabondage sexuel et on perçoit les femmes comme des "réservoirs" de maladie et de corruption ${ }^{53}$. À la fin du $\mathrm{XIX}^{\mathrm{e}}$ siècle, les solutions eugéniques des formes non conventionnelles de sexualité féminine s'étaient diffusées bien au-delà des œuvres canoniques d'Émile Zola pour investir la culture populaire où même les opposants à la moralité traditionaliste ont fini par se les approprier ${ }^{54}$.

Les effets de la diffusion de ce discours médical ne furent pas seulement conceptuels. Au XIXe siècle, sous l'influence du mouvement écossais Child-Saving, les jeunes garçons placés en institution étaient suivis pour leurs tendances criminelles et les jeunes filles pour la précocité sexuelle «vicieuse » dont leur corps portait les traces $^{55}$. Au XXe siècle, au Canada et aux États-Unis, les institutions pour jeunes délinquants associent jugements moraux et médicaux

\footnotetext{
51 Manderson 1997 ; Smith 2004.

52 Donzelot 1977.

53 Ganev 2007.

54 Emery 1994 ; Downing 2009 ; Robb 1996.

55 Mahood \& Littlewood 1994.
} 
dans les étiquettes ("psychopathe», "hystérique ») attribuées aux jeunes filles suspectées de vagabondage sexuel - pêché capital pour toute jeune fille, en adéquation avec l'obsession chrétienne de la sexualisation du corps féminin ${ }^{56}$. La sexualité des femmes volages fut volontiers assimilée aux désordres sociaux de la vie urbaine, mais aussi aux déplacements de population dus à l'industrialisation rapide, ainsi qu'aux efforts des sociétés d'après-guerre pour rétablir les normes genrées traditionnelles.

Après la Première Guerre mondiale, l'Écosse réalisa une campagne de santé publique contre les maladies vénériennes que Roger Davidson décrit comme l'exemple type de ce mélange de perspectives morales et médicales. On assimilait culpabilité morale et dangerosité; on jugeait le vagabondage sexuel des «filles à problèmes » à l'aune de la menace qu'elles représentaient pour la santé de la "race» mais aussi en fonction de leur éventuelle réhabilitation morale. C'est précisément cette combinaison de morale sexuelle conservatrice et d'interventionnisme médical qui déboucha sur le refus inattendu de la part des fonctionnaires écossais de la santé publique de distribuer des préservatifs au prétexte que cela reviendrait à légitimer l'existence de relations sexuelles «irrégulières» tout en "promouvant activement l'immoralité puisqu'on supprimait ainsi les sanctions naturelles associées au vagabondage sexuel $\aleph^{57}$. Malgré ses attributs d'autorité scientifique, la médecine ne s'était donc pas non plus totalement libérée des croyances consolatrices de la culture populaire. Davidson nous dit également que les agressions sexuelles de jeunes filles étaient nombreuses dans l'Écosse du début du $\mathrm{XX}^{\mathrm{e}}$ siècle, d'autant plus qu'il était communément admis que les relations sexuelles avec une vierge permettaient de soigner les maladies vénériennes. Les experts médicaux avaient beau s’indigner collectivement de ces croyances erronées, il leur arrivait souvent de mettre en avant des raisons médicales résultant d'une mauvaise hygiène pour ne pas diagnostiquer une infection vénérienne quand ils

56 Sangster 1996 ; Knupfer 2000.

57 Davidson 1994 : 271. 
examinaient des jeunes filles issues des classes sociales les plus pauvres $^{58}$.

Une dernière série de thématiques tourne autour de la sexualité, du genre et de l'identité de l'État lui-même. Les recherches de Roger Davidson sur l'Écosse du XXe siècle révèlent que l'élite politique se sentait concernée par la sexualité de ses citoyennes et citoyens et qu'elle s'est mobilisée pour intervenir dans la vie privée sexuelle de ses concitoyens. Les historiens de la monarchie de l'époque médiévale et de l'époque moderne savent depuis longtemps que la sexualité ou l'image genrée de la personne royale avait des répercussions non seulement sur l'image de la Nation à l'étranger, mais aussi sur la bonne ou mauvaise fortune du monarque au sein de son pays ${ }^{59}$. Cependant, c'est au moment de la Révolution française que genre et sexualité ont été perçus pour la première fois comme des métaphores de la Nation où le citoyen moyen est un avatar. Comme l'écrit Edmund Burke dans Reflections on the Revolution in France (1790), en Angleterre, la liberté politique menaçante de la Révolution française était couramment associée à la débauche sexuelle, ce qui donna lieu à des spéculations hostiles à la sexualité des féministes de la première vague, telle Mary Wollstonecraft, mais qui permit aussi de mettre en œuvre une législation qui, cherchant à consolider le mariage, réinscrivait le corps de la femme dans la passivité sexuelle ${ }^{60}$.

Ce genre de vent de panique nationale, tel que la Grande-Bretagne a pu alors en connaitre, a pris différentes formes. Dans certains cas, on a mis en question la masculinité des leaders hommes; dans d'autres cas, la peur généralisée de l'homosexualité a suscité des inquiétudes quant à la détermination des citoyens à partir au combat ${ }^{61}$. Mais on a également incriminé le comportement sexuel des femmes. La «culture de l'endiguement» du nouvel État libre d'Irlande, qui décréta l'alliance de l'État avec l'Église catholique, tenait les femmes

\footnotetext{
58 Davidson 2001.

59 Merrick 1990 ; Crawford 2003 ; Auslander 2000.

60 Binhammer 1996.

61 Voir Potter 2006 pour les interrogations concernant le directeur du F.B.I J. Edgar Hoover ; pour l'Allemagne avant la Première Guerre mondiale, voir Hull 2004.
} 
pour responsables de la réputation de la Nation. Tout un réseau de couvents de sœurs Madeleines et d'agences d'adoption isolait puis séparait les mères de leurs enfants illégitimes; les fonctionnaires fermaient les yeux ou ne faisaient pas grand cas des viols, incestes ou autres abus sexuels; ils n'enquêtaient pas plus sur les causes de la prostitution $^{62}$. De la même façon, de l'autre côté de la frontière, en Irlande du Nord, les femmes qui se sont laissé courtiser par les soldats américains stationnés là pendant la Seconde Guerre mondiale, se voyaient immanquablement reprocher tout désordre sexuel au nom d'une «identité communautaire» qui «appréhendait les actes de transgression de ces militaires à travers le prisme de la responsabilité des femmes $»^{63}$. Aujourd'hui, nous savons bien que les débats qui ont lieu au sein des États-Nations sur les conceptions divergentes de l'image et du comportement des femmes impliquent des questions politiques plus vastes où les femmes ne représentent qu'une part du problème (ou de la solution) ${ }^{64}$. Face aux interrogations sur le genre et les transgressions sexuelles, les Empires contemporains déclinants peuvent se révéler particulièrement vulnérables alors que des États plus unis s'avèrent plus à même d'affronter ces incertitudes ${ }^{65}$.

\section{Méthodes}

Les universitaires français ne s'étonnent plus de l'empressement avec lequel les historiens anglo-saxons ainsi que les spécialistes des études culturelles se sont emparés des idées de Michel Foucault pour étudier l'histoire de la sexualité. Foucault a souvent tourné en dérision les «faits» de l'histoire sociale; il n'eut de cesse de rechercher les vérités de l'auto-construction dans les formes plurivalentes de discours qui circulaient dans les réseaux de pouvoir entre " experts », dans les écrits qui amplifiaient et prolongeaient ces discours, mais aussi chez les individus et chez les groupes qui assimilaient,

62 Smith 2004.

63 McCormick 2006.

64 Frink 2009; pour la panique autour de l'inceste et du syndrome de la "lost memory", voir Satter 2003; sur la panique autour du mariage gay, voir Adam 2003.

65 Maxwell 2005 ; Demirci \& Somel 2008. 
transformaient ou encore résistaient à ces discours. Nous avons vu que les questions sur l'identité ou sur l'individualité sexuelle sont au premier plan dans les études anglo-américaines consacrées à la sexualité, surtout quand ces questions touchent les manifestations historiques des pratiques et des désirs homosexuels. Dans la mesure où la méthode historique est intrinsèquement opportuniste, les universitaires anglo-américains ont emprunté tels ou tels éléments théoriques à Foucault et à ses disciples, puis ils ont essayé d'établir les limites d'un paradigme foucaldien, avant de procéder à un amalgame entre son approche et celle de nombreux autres théoriciens. Foucault a très souvent été accusé de traiter les formes de l'identité sexuelle moderne comme les constructions d'un discours médical privant les individus de leur capacité d'agir (agency) sexuelle ${ }^{66}$, même si de telles accusations ont elles-mêmes été tout aussi vigoureusement démenties ${ }^{67}$.

Il reste difficile de cerner avec précision le véritable sujet de l'histoire de la sexualité. Les documents à exploiter sont pléthoriques : procès et textes juridiques, comportements sexuels rapportés par les individus eux-mêmes ou par des observateurs, études de cas médicaux et scientifiques, représentations médiatiques, artistiques ou fictionnelles, mémoires et confessions. Si nous aspirons à connaître la vérité sur les sexualités individuelles, il est néanmoins toujours possible d'écrire l'histoire sexuelle de personnages publics sans pour autant connaitre avec certitude leurs orientations et goûts en matière de sexualité68. En effet, même quand nous réussissons à connaître les pratiques des uns et des autres, nous avons souvent du mal à savoir ce que les gens ont ressenti dans ces moments-là et nous avons encore plus de difficulté à découvrir le pourquoi de leurs comportements, que l'on s'appuie sur le témoignage des intéressés ou sur d'autres témoignages. Nous devrions nous satisfaire de ces limites. Foucault nous a appris que la quête d'une sexualité "authentique" était vaine du fait que nous devons historiciser

\footnotetext{
66 Dixon 1997.

67 Boyarin \& Castelli 2001 : 365-366.

68 Potter 2006.
} 
minutieusement la quête d'une telle authenticité mais aussi les règles qui établissent cette même authenticité.

Le JHS a donné l'occasion aux chercheurs de soulever ces questions en fonction de leurs propres orientations. Mais ses rédacteurs en chef ont aussi fait preuve d'un grand professionnalisme en prêtant une réelle attention aux documents exploités et aux hypothèses avancées. Ils ont publié un numéro thématique très utile sur les méthodes employées dans le domaine. Julian Carter a ainsi souligné deux difficultés auxquelles les historiens des sexualités sont confrontés. La première concerne la tendance à reproduire les catégories culturelles ou juridiques de la déviance sexuelle et de sa répression du fait d'une lecture trop littérale des documents d'archives, ce qui peut aboutir à la réification des hiérarchies de pouvoir qui traversent sexe, genre et sexualité. La seconde difficulté est liée à cette téléologie qui conduit les historiens à rechercher les "premières" traces authentiques de ce qui, par la suite, est devenu une forme "moderne" d'identité sexuelle. Cette erreur fausse le passé et empêche de reconnaittre le très grand nombre de variations d'identités sexuelles et de désirs sexuels, qui sont rassemblées sous nos rubriques sexuelles contemporaines ${ }^{69}$. L'application de ces règles, simples en apparence, se complique lorsque les historiens persistent dans leur volonté d'atteindre la "vérité" de la sexualité en se demandant: que faisaient les gens et pourquoi l'ont-ils fait, qui étaient-ils et que croyaient-ils faire à l'époque?

Anna Clark conseille plutôt aux historiens de rechercher ces «moments crépusculaires» pendant lesquels comportement et désir échappent aux identités particulières pour se dévoiler selon l'instant, le lieu, les occasions et, vraisemblablement, selon les penchants de $\operatorname{chacun}^{70}$. Ce conseil est peut-être plus judicieux pour aborder les sexualités pré-modernes, quand les questions d'identité n'étaient pas encore des obsessions communautaires et individuelles. S'agissant des questions juridiques et de la répression de la sexualité "déviante", Stephen Robertson signale plusieurs choses : les relations complexes entre différentes catégories juridiques qui évoluent elles-mêmes; les

\footnotetext{
69 Carter 2005.

70 Clark 2005.
} 
contraintes de la déposition et des règles du témoignage; les conceptions évolutives de la maturité et de la responsabilité personnelle ; enfin, l'interaction entre loi, opinion publique et attitude du jury. Il souligne que ce genre d'analyse révèle la contingence historique de la loi et de ses applications, mais elle ne diminue pas la capacité de la loi à brutaliser, à rabaisser ou encore à humilier les individus dont le comportement ou l'image sont criminalisés ${ }^{71}$. Enfin, le sociologue et historien Jeffrey Weeks vient conforter les apports de Michel Foucault. Il insiste sur la façon dont Foucault a montré tous les paradoxes des identités sexuelles. Celles-ci avaient une fixité qui confirmait la non-fixité. Elles étaient à la fois sociales et personnelles, historiques et contingentes; elles étaient fictionnelles mais nécessaires ${ }^{72}$.

Je pourrais détailler encore les domaines d'investigation, les thèmes et les méthodes du JHS, mais peut-être la brève présentation que j’ai pu faire ici suffira-t-elle pour que les lecteurs français voient à quel point leurs travaux diffèrent, mais s'imbriquent aussi, dans les études consacrées à l'histoire de la sexualité telle qu'elle est représentée dans le JHS. Même si la revue n'est pas parvenue à évoquer toutes les sociétés historiques ni toutes les sexualités du monde, elle a réussi à mobiliser différentes perspectives disciplinaires dans un cadre qui éclaire les évolutions des sexualités humaines au fil du temps ainsi que la diversité des sexualités au travers de l'espace géographique. Des recherches issues de traditions différentes peuvent apprendre beaucoup les unes des autres sans que cela altère leurs perspectives propres. Ce qui est inacceptable, c'est que ces mêmes recherches s'ignorent; ironiquement, la domination impériale de la langue anglaise joue là un rôle qui est plus dangereux pour les puissances impériales que pour le reste du monde.

Traduction Isabelle Marquez, avec l'aide de Florence Cabaret (ERIAC, Université de Rouen).

71 Robertson 2005.

72 Weeks 2005. 


\section{Bibliographie}

ADAm Barry D., 2003, "The defense of marriage Act and American Exceptionalism: The "Gay marriage" Panic in the United States ", JHS, 12, 2, p. 259-276.

Allen Ann Taylor, 1993, «Feminism, Venereal Diseases, and the State in Germany, 1890-1918», JHS, 4, 1, p. 27-50.

AMER Saher, 2009, « Medieval Arab Lesbians and Lesbian-Like Women », JHS, 18, 2 , p. 215-236.

Altman Dennis, 2001, Global Sex, Chicago, University of Chicago Press.

AuSLANDER Leora, 2000, "Le Genre de la nation : la recherche aux États-Unis », Clio, Histoire, Femmes et Sociétés, 12, p. 193-208.

BArco Cristian, 2008, "Producing Patriarchs: Male Sodomy and Gender in Early Modern Spain », JHS, 17, 3, p. 351-376.

BAUER Heike, 2009, "Theorizing Female Inversion: Sexology, Discipline and Gender at the Fin-de-Siècle », JHS, 18, 1, p. 84-102.

Beccaloss Chiara, 2009, «The origin of Italian Sexological Studies: Female Sexual Inversion », JHS, 18, 2, p. 103-120.

BELL Shannon, 1995, "Pictures Don't Lie. Pictures Tell it all” ", JHS, 6, 2, p. 284 321.

BENNETT Judith M., 2000, "Lesbian-Like" and the Social History of Lesbianisms », JHS, 9, 1/2, p. 105-136.

BEREBITSKY Julie, 2006, "The Joy of Work: Helen Gurley Brown, Gender, and Sexuality in the White Collar Office », JHS, 15, 1, p. 89-127.

Binhammer Katherine, 1996, "The Sex Panic of the 1790s », JHS, 6, 3, p. 409-434.

Bitel Lisa, 1992, "Conceived in Sins, Born in Delights": Stories of Procreation from Early Ireland », JHS, 3, 2, p. 181-202.

BLEys Rudi, 1996, The Geography of Perversion: Male-To-Male Sexual Behavior Outside the West and the Ethnographic Imagination, 1750-1918, New York, New York University Press.

BOYARIN Daniel, 1995, "Are there any Jews in "The History of Sexuality"? », JHS, 5, 3, p. 333-355.

BOyarin Daniel \& Elizabeth CASTELli, 2001, "Foucault's The History of Sexuality: The Fourth Volume or, A Field Left Fallow for Others to Till », JHS, 10, 3/4, p. 357-374.

Bullough Vern, 1994, Sex in the Bedroom, New York, Basic Books.

CARTER Julian, 2005, « Theory, Method Praxis », JHS, 14, 1/2, p. 1-9.

Clark Anna, 1996, "Anne Lister's Construction of Lesbian Identity », JHS, 7, 1, p. 23-50.

—, 2005, «Twilight Moments », JHS, 14, 1/2, p. 139-160. 
Clark Catherine, 2007, "Purgatory, Punishment, and the Discourse of Holy Widowhood in the High and Later Middle Ages », vol. 14, 2, p. 169-203.

Clark Elizabeth A., 1995, "Antifamilial Tendencies in Ancient Christianity ", JHS, 5, 3, p. 356-380.

COHLER Deborah, 2007, "Sapphism and Sedition: Producing female homosexuality in Interwar Great Britain », JHS, 16, 1, p. 68-94.

COLLARD Judith, 2006, «Spiral Women: Locating Lesbian Activism in New Zealand Feminist Art, 1975-1992», JHS, 15, 2, p. 292-320.

CRAFT-FAIRCHILD Catherine, 2006, «Sexual and textual Indeterminacy: EighteenthCentury England and Representations of Sapphism », JHS, 15, 3, p. 408-431.

Crawford Katherine B., 2003, «Love, Sodomy, and the Sexual Reputation of Henry III », JHS, 12, 4, p. 513-542.

CRYLE Peter, 2009, “A Terrible Ordeal from every Point of View”: (Not) Managing Female Sexuality on the Wedding Night », JHS, 18, 1, p. 44-64.

Cryle Peter \& Lisa DownIng, 2009, «Female Sexual Pathologies », JHS, 18, 1, p. 1 7.

Davidson Roger, 1994, «Venereal Disease, Sexual Morality, and Public Health in Interwar Scotland », JHS, 5, 2, p. 267-294.

—, 2001, "“This Pernicious Delusion”: Law, Medicine, and Child Sexual Abuse in Early Twentieth-Century Scotland », JHS, 10, 1, p. 78-116.

Demirci Tuba \& Selçuk Somel, 2008, « Women’s Bodies, Demography, and Public Health: Abortion Policy and Perspectives in the Ottoman Empire of the Nineteenth Century », JHS, 17, 3, p. 377-420.

Dixon Joy, 1997, "Sexology and the Occult: Sexuality and Subjectivity in Theosophy's New Age », JHS, 7, 3, p. 409-433.

DonZelot Jacques, 1977, La Police des familles, Paris, Minuit.

DownING Lisa, 2009, « Murder in the Feminine: Marie Lafarge and the Sexualization of the Nineteenth-Century Criminal Woman », JHS, 18, 1, p. 121-137.

EDER Franz \& Lesley HaLl (eds), 1999, Sexual Cultures in Modern Europe, Vol. I, Manchester, Manchester University Press.

Elias Megan, 2006, " "Model Mamas": The domestic Partnership of Home Economics Pioneers Flora Rose and Martha Van Rensselaer », vol. 15, 1, p. 65 88.

EMERY Julie, 1994, «Steers, Queers, and Manifest Destiny: Representing the Lesbian Subject in Turn-of-the Century Texas », JHS, 5, 1, p. 26-57.

Erber Nancy, 1996, "The French Trials of Oscar Wilde », JHS, 6, 4, p. 549-588.

FASSIN Eric, 2004, "Le Genre aux États-Unis», in Christine BARD, Christian Baudelot \& Janine Mossuz-Lavau (éd.), Quand les femmes s'en mêlent. Genre et pouvoir, Paris, La Martinière, p. 23-43. 
Forman Ross G., 2002, "Randy on the Rand: Portuguese African Labor and the Discourse in "Unnatural Vice" in the Transvaal in the Early Twentieth Century ", JHS, 11, 4, p. 570-609.

Foucault Michel, 1986, The Use of Pleasure, transl. Robert Hurley, New York [traduction américaine de L'Histoire de la sexualité, tome II : L'Usage des plaisirs, Paris, Gallimard, 1984].

—, 1988, "Critical Theory/Intellectual History », in Lawrence KRITZMAN (ed.), Michel Foucault: Politics, Philosophy, Culture, New York [Version anglaise de «Structuralisme et post-structuralisme », entretien avec G. Raulet, Telos, vol. XVI, 55, printemps 1983, p. 195-211. Réédité dans Michel Foucault, 1994, Dits et écrits 1954-1988, tome IV : 1980-1988, édition établie sous la direction de Daniel DEFERT \& François EWALD avec la collaboration de Jacques LAGRANGE, Paris, Gallimard, 1994, p. 431-457].

FOUT John, 1990, «A Note from the Editor », JHS, 1, 1, p. 1.

FreEman Susan K., 2000, «From the Lesbian Nation to the Cincinnati Lesbian Community: Moving Toward a Politics of Location », JHS, 9, 1/2, p. 137-174.

FrINK Sandra, 2009, «Women, the Family, and the Fate of the Nation in American Anti-Catholic Narratives, 1830-1860 », JHS, 18, 2, p. 237-264.

GABBERT Ann R., 2003, "Prostitution and Moral Reform in the Borderlands: El Paso, 1890-1920», JHS, 12, 4, p. 575-604.

GANEV Robin, 2007, "Milkmaids, Ploughmen, and Sex in Eighteenth-Century Britain », JHS, 16, 1, p. 40-67.

GiLmore Leigh, 1994, «Obscenity, Modernity, Identity: Legalizing The Well of Loneliness and Nightwood», JHS, 4, 4, p. 603-624.

GiLmore Stephanie \& Elizabeth KAMINSKI, 2007, «A Part and Apart: Lesbian and Straight Feminist Activists Negotiate Identity in a Second-Wave Organization », JHS, 16, 1, p. 95-113.

Gordon Terri J., 2002, "Fascism and the Female Form: Performance Art in the Third Reich », JHS, 11, 1/2, p. 164-200.

Guereña Jean-Louis, 2008, "Prostitution and the Origins of the Governmental Regulatory System in Nineteenth-Century Spain ", JHS, 17, 2, p. 216-234.

Gwilliam Tassie, 1996, «Female Fraud: Counterfeit Maidenheads in the Eighteenth Century », JHS, 6, 4, p. 518-548.

Hannah-Jones Avril, 2003, "Competing Claims for Justice: Sexuality and Race at the Eighth Assembly of the Uniting Church in Australia, 1997 », JHS, 12, 2, p. 277-304.

Heinemann Elizabeth D., 2002, «Sexuality and Nazism: The Doubly Unspeakable? », JHS, 11, 1/2, p. 22-66.

Herzog Dagmar, 2002, «Hubris and Hypocrisy, Incitement and Disavowal: Sexuality and German Fascism », JHS, 11, 1/2, p. 3-22. 
Hogeland Lisa, 1995, "Sexuality in the Consciousness-Raising Novel of the 1970s », JHS, 5, 4, p. 601-632.

Hull Isabel, 2004, The Entourage of Kaiser Wilhelm II, Cambridge, Cambridge University Press.

IRvine Janice M., 1995, "Reinventing Perversion: Sex Addiction and Cultural Anxieties », JHS, 5, 3, p. 429-450.

JACKSON Peter A., 1997, "Thai Research on Male Homosexuality and the Cultural Limits of Foucaultian Analysis », JHS, 8, 1, p. 52-85.

KAMPF Antje, 2008, "Controlling Male Sexuality: Combating Venereal Disease in the New Zealand Military during Two World Wars », JHS, 17, 2, p. 235-258.

Karras Ruth, 1990, "Holy Harlots: Prostitute Saints in Medieval Legend », JHS, 1, 1, p. 3-32.

Knupfer Anne Meis, 2000, "“To Become Good, Self-Supporting Women”: The State Industrial School for Delinquent Girls at Geneva, Illinois, 1900-1935 », JHS, 9, 4, p. 420-446.

KraAkman Dorelies, 1994, "Reading Pornography Anew: A Critical History of Sexual Knowledge for Girls in French Erotic Fiction, 1750-1840», JHS, 4, 4, p. 517-548.

LEIDINGER Christiane, 2004, «Anna Rüling: A Problematic Foremother of Lesbian History ", JHS, 13, 4, p. 477-499.

LEVINE Philippa, 1994, «Venereal Disease, Prostitution, and the Politics of Empire: The Case of British India », JHS, 4, 4, p. 579-602.

MAHOOD Linda \& Barbara LiTTLEWOOD, 1994, "The "Vicious" Girl and the "Street-Corner" Boy: Sexuality and the Gendered Delinquent in the Scottish Child-Saving Movement, 1850-1940», JHS, 4, 4, p. 549-578.

MANDERSON Lenore, 1997, "Colonial Desires: Sexuality, Rape, and Gender in British Malaya », JHS, 7, 3, p. 372-388.

MAtysik Tracy, 2004, "In the Name of the Law: The "Female Homosexual" and the Criminal Code in Fin-de-Siècle Germany », JHS, 13, 1, p. 26-48.

MAXWELL Alexander, 2005, «Sexual Stereotypes in the Habsburg Empire », JHS, 14, 3, p. 266-290.

MCCORMick Leanne, 2006, "One Yank and They're Off: Interaction between U.S. Troops and Northern Irish Women, 1942-1945», JHS, 15, 2, p. 228-257.

MEEM Deborah T., 1997, «Eliza Lynn Linton and the Rise of Lesbian Consciousness », JHS, 7, 4, p. 537-560.

Merrick Jeffrey, 1990, "Sexual Politics and Public Order in Late EighteenthCentury France: The Mémoires secrets and the Correspondance secrète», JHS, 1, 1, p. 68-85.

MESCH Rachel, 2009, "Housewife or Harlot?": Sex and the Married Woman in Nineteenth-Century France », JHS, 18, 1, p. 65-83. 
Moeller Robert G., 1994, ““'The Homosexual Man' is a 'Man,' the 'Homosexual Woman' is a 'Woman"': Sex Society and the Law in Postwar West Germany ", JHS, 4, 3, p. 395-429.

MoOre Alison, 2009, «Rethinking Gendered Perversion and Degeneration in Visions of Sadism and Masochism », JHS, 18, 1, p. 138-157.

Murray Heather, 2007, "Free for all Lesbians: Lesbian Cultural Production and Consumption in the United states during the 1970s », JHS, 14, 2, p. 251-275.

NeuHaus Jessamyn, 2000, «The Importance of Being Orgasmic: Sexuality, Gender, and Marital Sex Manuals in the United States, 1920-1963 ", JHS, 9, 4, p. 447-473.

Nye Robert A., 2003, "The Pacte Civil de Solidarité and the History of Sexuality ", French Politics, Society and Culture, vol. 21, 1, p. 87-100.

- 2008, "The Biosexual Foundations of Our Modern Concept of Gender », in Nicole C. Karafyllis \& Gotlund UlshÖFER (eds.), Sexualized Brains: Scientific Modeling of Emotional Intelligence from a Cultural Perspective, Cambridge, MA, MIT University Press, p. 68-81.

O'ConNor Erin, 1995, «Pictures of Health: Medical Photography and the Emergence of Anorexia Nervosa», JHS, 5, 4, p. 535-572.

OFFEN Karen, 2006, "Le gender est-il une invention américaine?", Clio, Histoire, Femmes et Sociétés, 24, p. 291-304.

Penrose Walter, 2001, «Hidden in History: Female Homoeroticism and Women of a "Third Nature" in the South Asian Past », JHS, 10, 1, p. 3-39.

PHIPPS Pauline, 2009, «Faith, Desire, and Sexual Identity: Constance Maynard's Atonement for Passion », JHS, 18, 2, p. 265-286.

Pitzulo Carrie, 2008, «The Battle in Every Man's Bed: Playboy and the Fiery Feminists ", JHS, 17, 2, p. 259-289.

PotTer Claire B., 2006, «Queer Hoover: Sex, Lies, and Political History », JHS, 15, 3, p. 355-381.

Proschan Frank, 2002, "Syphilis, Opiomania, and Pederasty": Colonial Constructions of Vietnamese (and French) Social Diseases ", JHS, 11, 4, p. 610636.

REBREYEND Anne-Claire, 2005, "Comment écrire l'histoire des sexualités au XX ${ }^{\mathrm{e}}$ siècle ? ", Clio, Histoire, Femmes et Sociétés, 22, p. 185-209.

Richlin Amy, 1993, «Not Before Homosexuality: The Materiality of the Cinaedus and the Roman Law against Love between Men », JHS, 3, 4, p. 523-573.

RIVERS Christopher, 1995, «Safe Sex: The prophylactic Walls of the Cloister in the French Libertine Convent Novel of the Eighteenth Century », JHS, 5, 3, p. 381402.

RoBB George, 1996, "The Way of All Flesh: Degeneration, Eugenics, and the Gospel of Free Love », JHS, 6, 4, p. 589-603. 
ROBERTSON Stephen, 2005, «What's Law Got to do with it? Legal Records and Sexual Histories », JHS, 14, 1/2, p. 161-185.

RobINSON Paul, 1976, The Modernization of Sex, New York, Harper \& Row.

Rupp Leila, 2001, "Toward a Global History of Same-Sex Sexuality », JHS, 10, 2, p. 287-302.

SANGSTER Joan, 1996, "Incarcerating "Bad Girls": The regulation of Sexuality through the Female Refuges Act in Ontario, 1920-1945 », JHS, 7, 2, p. 239-275.

SATTER Beryl, 2003, «The Sexual Abuse Paradigm in Historical Perspective: Passivity and Emotion in Mid-Twentieth-Century America », JHS, 12, 3, p. 424-464.

SCOTT Joan, 1988, Gender and the Politics of History, New York, Columbia University Press.

SHARP Ingrid, 2004, "The Sexual Unification of Germany », JHS, 13, 3, p. 348-365.

Sigal Pete, 1997, "The Politicization of Pederasty among the Colonial Yucatecan Maya », JHS, 8, 1, p. 1-24.

SMith James M., 2004, "The Origins of Ireland's Containment Culture and the Corrigan Report », JHS, 13, 2, p. 208-233.

Smith-Rosenberg Carroll, 1985, Disorderly Conduct: Visions of Gender in Victorian America, New York, Alfred A. Knopf.

Spaulding Jay \& Stephanie Beswick, 1995, «Sex, Bondage and the Market: The Emergence of Prostitution in Northern Sudan, 1750-1950», JHS, 5, 4, p. 512 535.

SpongBerg Mary, 1998, Feminizing Venereal Disease: The Body of the prostitute in Nineteenth-Century Medical Discourse, New York, New York University Press.

SweET Michael J. \& Leonard Zwilling, 1993, "The First Medicalization: The Taxonomy and Etiology of Queerness in Classical Indian Medicine », JHS, 3, 4, p. 590-607.

TAYLOR Rabun, 1997, "Two Pathic Subcultures in Ancient Rome », JHS, 7, 3, p. 319-371.

ThÉBAud Françoise \& Michelle ZANCARINI-Fournel, 2002, « Naissance et histoire d'une revue ", Clio, Histoire, Femmes et Sociétés, 16, p. 9-22.

Tiefer Lenore, 1995, Sex is Not a Natural Act and Other Essays, Boulder, Colorado, Westview.

Timm Annette F., 2002, «Sex with a Purpose: Prostitution, Venereal Disease, and Militarized Masculinity in the Third Reich », JHS, 11, 1/2, p. 223-256.

WEEKS Jeffrey, 2005, « Remembering Foucault », JHS, 14, 1/2, p. 186-201.

Wertheimer Laura, 2006, «Children of Disorder: Clerical Parentage, Illegitimacy, and reform in the Middle Ages », JHS, 15, 3, p. 382-407.

WORMAN Caroline, 2009, «From Lamarck to Aberrations: Nature, Hierarchies, and Gender », JHS, 18, 1, p. 8-25. 